University of Wollongong

Research Online

Faculty of Engineering - Papers (Archive)

Faculty of Engineering and Information

Sciences

2011

\title{
Visualization of vortex motion in FeAs-based BaFe1.9Ni0.1As2 single crystal by means of magneto-optical imaging
}

Zhi W. Lin

Univ Technol Sydney

Yong Jian Li

Univ of Technology Sydney

Jian G. Zhu

Univ Technol Sydney

Xiaolin Wang

University of Wollongong, xiaolin@uow.edu.au

S. X. Dou

University of Wollongong, shi@uow.edu.au

See next page for additional authors

Follow this and additional works at: https://ro.uow.edu.au/engpapers

Part of the Engineering Commons

https://ro.uow.edu.au/engpapers/1345

\section{Recommended Citation}

Lin, Zhi W.; Li, Yong Jian; Zhu, Jian G.; Wang, Xiaolin; Dou, S. X.; Guo, Youguang; Lei, Gang; Wang, Yi; Philips, Matthew; Cortie, Michael; Li, Yan-Cheng; Choi, Ki-Young; and Shi, Xun: Visualization of vortex motion in FeAs-based BaFe1.9Ni0.1As2 single crystal by means of magneto-optical imaging 2011. https://ro.uow.edu.au/engpapers/1345 


\section{Authors}

Zhi W. Lin, Yong Jian Li, Jian G. Zhu, Xiaolin Wang, S. X. Dou, Youguang Guo, Gang Lei, Yi Wang, Matthew Philips, Michael Cortie, Yan-Cheng Li, Ki-Young Choi, and Xun Shi 


\title{
Visualization of vortex motion in FeAs-based $\mathrm{BaFe}_{1.9} \mathrm{Ni}_{0.1} \mathrm{As}_{2}$ single crystal by means of magneto-optical imaging
}

\author{
Zhi Wei Lin, ${ }^{1, a)}$ Yong Jian Li, ${ }^{1,2}$ Jian Guo Zhu, ${ }^{1}$ Xiao Lin Wang, ${ }^{3}$ Shi Xue Dou, ${ }^{3}$ \\ You Guang Guo, ${ }^{1}$ Gang Lei, ${ }^{1}$ Yi Wang, ${ }^{1}$ Matthew Philips, ${ }^{1}$ Michael Cortie, ${ }^{1}$ \\ Yan Cheng Li, ${ }^{1}$ Ki-Young Choi, ${ }^{4}$ and Xun $\mathrm{Shi}^{5}$ \\ ${ }^{1}$ University of Technology, Sydney, PO Box 123, Broadway, NSW 2007, Australia \\ ${ }^{2}$ Province-Ministry Joint Key Laboratory of Electromagnetic Field and Electrical Apparatus Reliability, \\ Hebei University of Technology, Tianjin 300130, People's Republic of China \\ ${ }^{3}$ Institute for Superconducting and Electronic Materials, University of Wollongong, Wollongong, \\ New South Wales 2522, Australia \\ ${ }^{4}$ School of Physics, Sogang University, Korea \\ ${ }^{5}$ Shanghai Institute of Ceramics, Shanghai, 200050 China
}

(Presented 16 November 2010; received 5 October 2010; accepted 8 December 2010; published online 6 April 2011; publisher error corrected 14 April 2011)

\begin{abstract}
Superconductivity has been found in newly discovered iron-based compounds. This paper studies the motion of magnetic vortices in $\mathrm{BaFe}_{1.9} \mathrm{Ni}_{0.1} \mathrm{As}_{2}$ single crystal by means of the magneto-optical imaging technique. A series of magneto-optical images reflecting magnetic flux distribution at the crystal surface were taken when the crystal was zero-field cooled to $10 \mathrm{~K}$. The behavior of the vortices, including penetration into and expulsion from the single crystal with increasing and decreasing external fields, respectively, is discussed. The motion behavior is similar to that observed in high- $T_{\mathrm{c}}$ superconducting cuprates with strong vortex pinning; however, the flux-front is irregular due to randomly distributed defects in the crystal. (C) 2011 American Institute of Physics. [doi:10.1063/1.3562261]
\end{abstract}

\section{INTRODUCTION}

The discovery of superconductivity in the oxypnictides phase, $\mathrm{LaFe}(\mathrm{OF}) \mathrm{As},{ }^{1,2}$ has stimulated strong interest in understanding the physical properties of the new Fe-based superconducting materials, mechanisms of superconductivity, and differences between the Fe-based superconducting compounds from the high- $T_{\mathrm{c}}$ cuprates. Superconductivity has been found in a wide variety of compounds with $\mathrm{Fe}^{2+}$ square planar sheets, such as the oxypnictides of $\mathrm{LaFeO}_{1-x} \mathrm{~F}_{x} \mathrm{As}$ (or $\mathrm{LaFeO}_{1-x} \mathrm{As}$ ) and doped $\mathrm{ThCr}_{2} \mathrm{Si}_{2}$-type structure compounds of $\mathrm{BaFe}_{2} \mathrm{As}_{2},{ }^{3} \mathrm{LiFeAs},{ }^{4}$ and $\mathrm{FeSe} .{ }^{5-7}$ It also has been noticed that superconductivity can be induced in $\mathrm{BaFe}_{2} \mathrm{As}_{2}$ by alloying $\mathrm{Fe}$ with the other ferromagnetic $3 \mathrm{~d}$ elements, such as $\mathrm{Co}$ (Ref. 8) or $\mathrm{Ni}^{9,}{ }^{9,10}$ similar to the oxypnictides ${ }^{11,12}$ and (Ba,K)$\mathrm{Fe}_{2} \mathrm{As}_{2}{ }^{13}$ Investigation of the vortex properties in new $\mathrm{Fe}-$ based superconducting materials will provide a fundamental understanding of the materials. Magneto-optical imaging (MOI), as a simple and versatile method, can easily visualize the motion of local vortices in superconducting materials on the micrometer scale. ${ }^{14}$ In comparison, the superconducting quantum interference devices and the vibrating sample magnetometer normally study collective magnetic properties.

While vortex motion in Co-doped $\mathrm{Ba}\left(\mathrm{Fe}_{1.8} \mathrm{Co}_{0.2}\right) \mathrm{As}_{2}$ single crystal has been studied, ${ }^{15}$ this paper studies the properties of local magnetic vortices in Ni-doped $\mathrm{BaFe}_{1.9} \mathrm{Ni}_{0.1} \mathrm{As}_{2}$ single crystal by means of the MOI technique after the sample was zero-field cooled down to $10 \mathrm{~K}$. The observed

\footnotetext{
a) Author to whom correspondence should be addressed. Electronic mail: jacklin@eng.uts.edu.au.
}

motion of vortices was found to be very similar to that in high $T_{\mathrm{c}}$ cuprates with strong flux pinning strengths.

\section{EXPERIMENTAL DETAILS}

The nominal composition $\mathrm{Ba}\left(\mathrm{Fe}_{1.9} \mathrm{Ni}_{0.1}\right) \mathrm{As}_{2}$ single crystals were grown out of $\mathrm{Fe}-\mathrm{As}$ flux by the self-flux method, as used for $\mathrm{Ba}\left(\mathrm{Fe}_{1.8} \mathrm{Co}_{0.2}\right) \mathrm{As}_{2}$ single crystal. ${ }^{8}$ The size of the rectangular-shaped single crystal studied is $2.7 \times 1.8 \times 0.2$ $\mathrm{mm} .{ }^{3}$ The crystal is brittle, with well-formed plates with the [001] direction perpendicular to the plane of the crystal. However, the edges of the crystal and the corners are not well defined.

The magneto-optical images shown in the paper were captured using the MOI technique, ${ }^{16}$ which was built based on the Faraday rotation of polarized light in a Bi-doped iron garnet indicator film with in-plane magnetization. The optical cryostat was cooled by a compressor. The temperature can reach down to $10 \mathrm{~K}$, and the applied external field can be up to $17 \mathrm{kOe}$. The indicator film was placed directly on top of the crystal with slight mechanical pressure to ensure that the field distribution at the crystal surface could be precisely imaged by the indicator film.

\section{RESULTS AND DISCUSSION}

Figure 1 shows the temperature dependence of electrical resistivity in the $a-b$ plane in the absence of magnetic field. Measurements were performed on a Quantum Design Physical Property Measurement System. The crystal shows metallic behavior at temperatures above the transition temperature. The resistivity drops down abruptly at $18.8 \mathrm{~K}$ and reaches zero at 


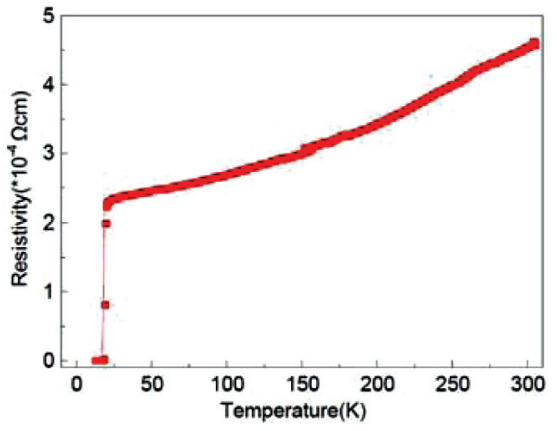

FIG. 1. (Color online) Temperature dependence of resistivity.

$17.4 \mathrm{~K}$. The transition width is $1.4 \mathrm{~K}$. This value is larger than that reported for $\mathrm{Ba}\left(\mathrm{Fe}_{1.904} \mathrm{Ni}_{0.096}\right) \mathrm{As}_{2}$ (Ref. 17) (less than $1 \mathrm{~K}$ ) and for $\mathrm{Ba}\left(\mathrm{Fe}_{1.8} \mathrm{Co}_{0.2}\right) \mathrm{As}_{2}$ single crystal $(0.6 \mathrm{~K}){ }^{8}$

Figure 2 shows the magnetization hysteresis loop of the crystal measured at $5 \mathrm{~K}$ with an applied magnetic field along the $c$-crystallographic direction. It can be seen that the lower critical field $H_{c 1}$ is very small, around $50 \mathrm{Oe}$. Limited by the maximum field of $5 \mathrm{~T}$, the second peak for fishtail effect, as shown in $\mathrm{Ba}\left(\mathrm{Fe}_{1.8} \mathrm{Co}_{0.2}\right) \mathrm{As}_{2},{ }^{8}$ is not observed. It is believed that the fishtail ${ }^{18}$ effect could be observed at a higher applied field or at higher temperatures with the field up to $5 \mathrm{~T}$, as observed in $\mathrm{Ba}_{0.6} \mathrm{~K}_{0.4} \mathrm{Fe}_{2} \mathrm{As}_{2}$ single crystal. ${ }^{19}$

Figure 3 presents magneto-optical images taken at $10 \mathrm{~K}$ and with different applied magnetic fields parallel to the $c$ direction. The contrast of Fig. 3(a) was enhanced using image editing software. The tooth-pattern domains resulted from the stray field parallel to the indicator film. Some defects in the indicator film can be clearly seen in Figs. 3(d) and 3(e). At the fields below $50 \mathrm{Oe}$, the vortices were completely shielded from the crystal, and the crystal was in the Meissner state. As the field rose over $60 \mathrm{Oe}$, the vortices started penetrating into the crystal from points along the edge, as shown in Fig. 3(a), which was taken at 83 Oe. As the applied field increased further, the vortices penetrated further into the crystal. In addition, penetration also occurred along the crystal edges, as shown in Figs. 3(b) and 3(c). The behavior is very similar to that in high- $T_{\mathrm{c}}$ cuprates. At 531 Oe, the salient flux front reached the central region of the crystal, as shown in Fig. 3(d), but there was still a small fluxfree region. At about 584 Oe, the vortices occupied the whole crystal, and the flux-free region disappeared. The crystal was in a mixed state. Figure 3(e) is the magneto-

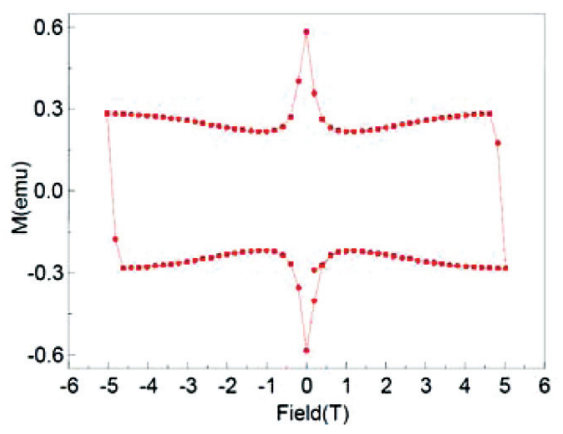

FIG. 2. (Color online) Magnetization hysteresis loops measured at $5 \mathrm{~K}$ with the fields along the $c$-crystallographic direction.
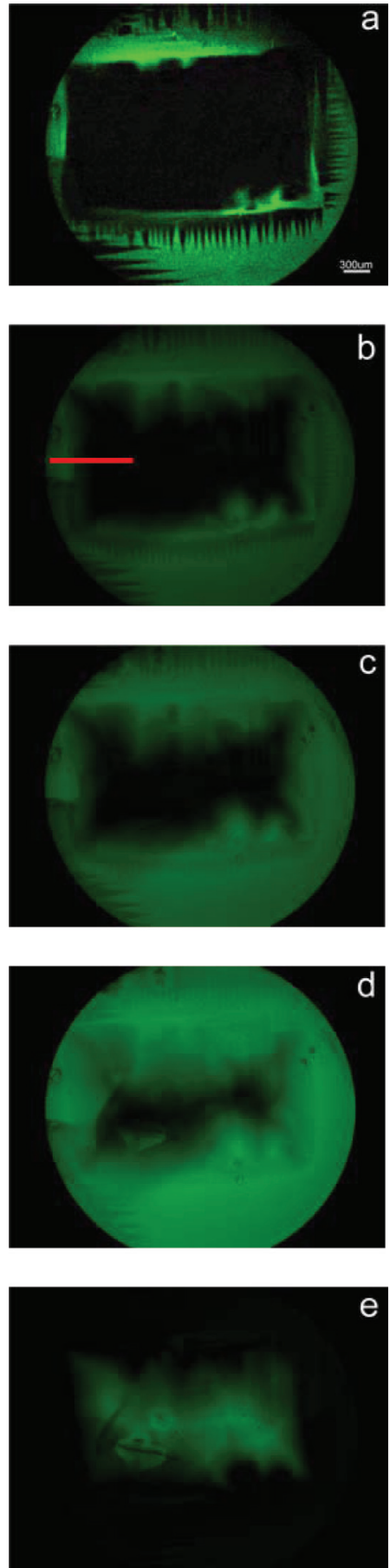

FIG. 3. (Color online) Magneto-optical images taken at $10 \mathrm{~K}$ and with applied magnetic fields of $83 \mathrm{Oe}$ (a), $265 \mathrm{Oe}$ (b), $354 \mathrm{Oe}$ (c), $531 \mathrm{Oe}$ (d), and in the remanent state (e).

optical image for the remanent state after the field increased to 1700 Oe. It shows clearly that the penetrated vortices are pinned in the central region of the crystal and the vortices around the crystal edges have left the crystal.

In order to clearly present the progress of the flux front moving in the crystal, Fig. 4 shows the superposition of fluxfree regions at different applied fields. The vortex penetration initially occurs at the defect points along the edges. As the field increases further, the existing vortices penetrate further into the crystal. In addition, vortex penetration occurs along the edge, forming irregularly shaped flux fronts. As a 


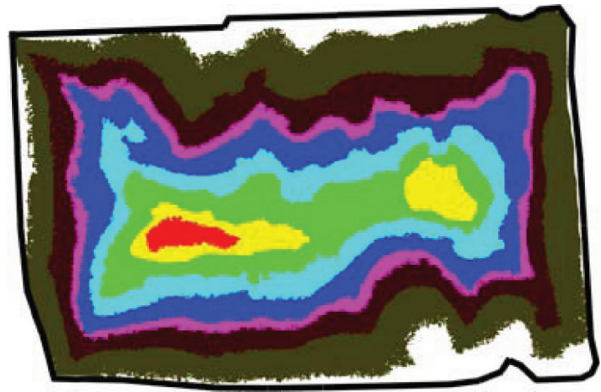

FIG. 4. (Color online) Flux-free regions for applied fields of 109 Oe, 135 Oe, 265 Oe, 299 Oe, 354 Oe, 430 Oe, 502 Oe, and 531 Oe. The outermost black line is the outline of the crystal.

result, the flux-free region shrinks continuously. This observation indicates that the crystal has strong vortex pinning, which damps the motion of the vortices. It can also be seen that the vortices penetrate into the crystal easily from the defect points along the edges, but these defect points are not the starting points of the channels along which the vortices can easily enter the crystal. Because these crystal structure defects are randomly distributed in the crystal, the flux-front shape is irregular.

The critical current density can be estimated using the Bean critical state model $^{20}$ as follows:

$$
\frac{d}{d x} B_{z}(x)=\mu_{0} J_{\mathrm{c}}
$$

According to the field distribution along the horizontal line shown in Fig. 3(b), the critical current density, $J_{\mathrm{c}}$, is estimated to be $5.5 \times 10^{5} \mathrm{~A} / \mathrm{cm}^{2}$. This value is similar to the value reported in unirradiated $\mathrm{Ba}\left(\mathrm{Fe}_{0.93} \mathrm{Ni}_{0.07}\right)_{2} \mathrm{As}_{2}$ crystal at $10 \mathrm{~K} .{ }^{21}$

\section{CONCLUSION}

The single crystal $\mathrm{Ba}\left(\mathrm{Fe}_{1.9} \mathrm{Ni}_{0.1}\right) \mathrm{As}_{2}$ was grown from $\mathrm{Fe}-\mathrm{As}$ flux and had a transition temperature of $18.8 \mathrm{~K}$ and transition width of $1.4 \mathrm{~K}$. The vortex motion in the crystal was studied by means of magneto-optical imaging with increasing and decreasing applied magnetic fields parallel to the $c$-direction after zero-field cooling. In general, the crystal is in the Meissner state at fields below the low critical field. As the applied field increases further, the vortices penetrate into the crystal along the edges over the low critical field and move toward the center of the crystal. While the field decreases to zero, the penetrated vortices are pinned in the crystal with the remanent state. Such behavior is very similar to the vortex motion in high- $T_{\mathrm{c}}$ cuprates with strong vortex pinning. However, an irregular flux-front shape was observed due to the structural defects randomly distributed in the crystal. The critical current density, $J_{\mathrm{c}}$, estimated from the flux distribution is about $5.5 \times 10^{5} \mathrm{~A} / \mathrm{cm}^{2}$.

\section{ACKNOWLEDGMENTS}

This work is supported by the Australian Research Council and University of Technology, Sydney, Australia.

${ }^{1}$ Y. Kamihara, T. Watanabe, M. Hirano, and H. Hosono, J. Am. Chem. Soc. 130, 3296 (2008).

${ }^{2}$ H. Takahashi, K. Igawa, K. Arii, Y. Kamihara, M. Hirano, and H. Hosono, Nature 453, 376 (2008).

${ }^{3}$ M. Rotter, M. Tegel, and D. Johrendt, Phys. Rev. Lett. 101, 107006 (2008).

${ }^{4}$ X. C. Wang, Q. Q. Liu, Y. X. Lv, W. B. Gao, L. X. Yang, R. C. Yu, F. Y. Li, and C. Q. Jin, Solid State Commun. 148, 538 (2008).

${ }^{5}$ F. C. Hsu, J. Y. Luo, K. W. Yeh, T. K. Chen, T. W. Huang, P. M. Wu, Y. C. Lee, Y. L. Huang, Y. Y. Chu, D. C. Yan, and M. K. Wu, Proc. Natl. Acad. Sci. U.S.A 105, 14262 (2008).

${ }^{6}$ W. D. Si, Z. W. Lin, Q. Jie, W. G. Yin, J. Zhou, G. D. Gu, P. D. Johnson, and Q. Li, Appl. Phys. Lett. 95, 3 (2009).

${ }^{7}$ J. Wen, G. Xu, Z. Xu, Z. W. Lin, Q. Li, W. Ratcliff, G. Gu, and J. M. Tranquada, Phys. Rev. B 80, 104506 (2009).

${ }^{8}$ A. S. Sefat, R. Jin, M. A. McGuire, B. C. Sales, D. J. Singh, and D. Mandrus, Phys. Rev. Lett. 101, 117004 (2008).

${ }^{9}$ L. J. Li, Y. K. Luo, Q. B. Wang, H. Chen, Z. Ren, Q. Tao, Y. K. Li, X. Lin, M. He, Z. W. Zhu, G. H. Cao, and Z. A. Xu, New J. Phys. 11, 8 (2009).

${ }^{10}$ A. S. Sefat, D. J. Singh, R. Jin, M. A. McGuire, B. C. Sales, and D. Mandrus, Phys. Rev. B 79, 024512 (2009).

${ }^{11}$ A. S. Sefat, A. Huq, M. A. McGuire, R. Jin, B. C. Sales, D. Mandrus, L. M. D. Cranswick, P. W. Stephens, and K. H. Stone, Phys. Rev. B 78, 104505 (2008).

${ }^{12}$ Z. Li, G. Chen, J. Dong, G. Li, W. Hu, D. Wu, S. Su, P. Zheng, T. Xiang, N. Wang, and J. Luo, Phys. Rev. B 78, 060504 (2008).

${ }^{13}$ X.-L. Wang, S. R. Ghorbani, S.-I. Lee, S. X. Dou, C. T. Lin, T. H. Johansen, K. H. Müller, Z. X. Cheng, G. Peleckis, M. Shabazi, A. J. Qviller, V. V. Yurchenko, G. L. Sun, and D. L. Sun, Phys. Rev. B 82, 024525 (2010).

${ }^{14}$ S. J. Bending, Adv. Phys. 48, 449 (1999).

${ }^{15}$ Z. W. Lin, J. Zhu, Y. Guo, Y. Li, S. Wang, Y. B. Zhang, K. X. Xu, and C. B. Cai, J. Appl. Phys. 107, 09E155 (2010).

${ }^{16}$ Z. W. Lin, J. W. Cochrane, G. J. Russell, S. X. Dou, and H. K. Liu, Supercond. Sci. Technol. 11, 1017 (1998).

${ }^{17}$ L. J. Li, Y. K. Luo, Q. B. Wang, H. Chen, Z. Ren, Q. Tao, Y. K. Li, X. Lin, M. He, Z. W. Zhu, G. H. Cao, and Z. A. Xu, New J. Phys. 11, 8 (2009).

${ }^{18}$ M. Jirsa, L. Půst, D. Dlouhý, and M. R. Koblischka, Phys. Rev. B 55, 3276 (1997).

${ }^{19}$ H. Yang, H. Luo, Z. Wang, and H.-H. Wen, Appl. Phys. Lett. 93, 142506 (2008).

${ }^{20}$ C. P. Bean, Rev. Mod. Phys. 36, 31 (1964).

${ }^{21}$ Y. Nakajima, Y. Tsuchiya, T. Taen, T. Tamegai, S. Okayasu, and M. Sasase, Phys. Rev. B 80, 012510 (2009). 